\title{
Evolution of Inbreeding Avoidance and Inbreeding Preference through Mate Choice among Interacting Relatives
}

\author{
A. Bradley Duthie* and Jane M. Reid \\ Institute of Biological and Environmental Sciences, School of Biological Sciences, Zoology Building, Tillydrone Avenue, University \\ of Aberdeen, Aberdeen AB24 2TZ, United Kingdom
}

Submitted March 29, 2016; Accepted July 28, 2016; Electronically published October 28, 2016

Online enhancements: appendixes, supplemental PDF. Dryad data: http://dx.doi.org/10.5061/dryad.3861f.

\begin{abstract}
Aвstract: While extensive population genetic theory predicts conditions favoring evolution of self-fertilization versus outcrossing, there is no analogous theory that predicts conditions favoring evolution of inbreeding avoidance or inbreeding preference enacted through mate choice given obligate biparental reproduction. Multiple interacting processes complicate the dynamics of alleles underlying such inbreeding strategies, including sexual conflict, distributions of kinship, genetic drift, purging of mutation load, direct costs, and restricted kin discrimination. We incorporated these processes into an individual-based model to predict conditions where selection should increase or decrease frequencies of alleles causing inbreeding avoidance or inbreeding preference when females or males controlled mating. Selection for inbreeding avoidance occurred given strong inbreeding depression when either sex chose mates, while selection for inbreeding preference occurred given very weak inbreeding depression when females chose but never occurred when males chose. Selection for both strategies was constrained by direct costs and restricted kin discrimination. Purging was negligible, but allele frequencies were strongly affected by drift in small populations, while selection for inbreeding avoidance was weak in larger populations because inbreeding risk decreased. Therefore, while selection sometimes favored alleles underlying inbreeding avoidance or preference, evolution of such strategies may be much more restricted and stochastic than is commonly presumed.
\end{abstract}

Keywords: inbreeding strategy, mate choice, mating system, reproductive strategy, relatedness, fitness.

\section{Introduction}

Inbreeding, defined as reproduction among relatives, can alter genotype frequencies and substantially affect individual fitness and, thereby, profoundly affect trait evolution and population persistence (Fisher 1949; Charlesworth and

\footnotetext{
* Corresponding author; e-mail: aduthie@abdn.ac.uk.

Am. Nat. 2016. Vol. 188, pp. 651-667. (C) 2016 by The University of Chicago. 0003-0147/2016/18806-56892\$15.00. All rights reserved. This work is licensed under a Creative Commons Attribution-NonCommercial 4.0 International License (CC BY-NC 4.0), which permits non-commercial reuse of the work with attribution. For commercial use, contact journalpermissions@press.uchicago.edu. DOI: $10.1086 / 688919$
}

Charlesworth 1987; Keller and Waller 2002; Theodorou and Couvet 2006; Angeloni et al. 2011; Karron et al. 2012; Wright et al. 2013). Understanding such evolutionary and ecological dynamics requires understanding the extent to which females and males avoid or prefer inbreeding through nonrandom mate choice and, therefore, of the ways in which evolving mating systems can themselves create inbreeding. Such understanding requires theoretical frameworks that predict the conditions under which active strategies of inbreeding avoidance or preference are themselves expected to evolve (Lande and Schemske 1985; Goodwillie et al. 2005; Cheptou and Donohue 2011; Szulkin et al. 2013).

Inbred offspring are typically less fit than outbred offspring, termed "inbreeding depression" (Charlesworth and Charlesworth 1999; Keller and Waller 2002; Charlesworth and Willis 2009). Consequently, animal ecologists frequently presume that reproductive strategies that reduce biparental (i.e., nonself) inbreeding will be adaptive and evolve (Pusey and Wolf 1996; Tregenza and Wedell 2002; Tregenza et al. 2006; Geffen et al. 2011; Szulkin et al. 2013; Tennenhouse 2014). But this presumption ignores a potential inclusive fitness benefit of inbreeding. In an outbreeding population, a focal individual that inbreeds increases its inclusive fitness because inbreeding increases the reproductive success of the focal individual or its related mate (depending on sex roles; Parker 1979, 2006). Given sufficiently weak inbreeding depression, a mutant allele that causes inbreeding is, therefore, expected to increase in frequency (Fisher 1941; Lande and Schemske 1985; Lloyd 1992; Szulkin et al. 2013). This transmission advantage underpins extensive theory predicting the evolution of outcrossing versus self-fertilization (Fisher 1941; Lande and Schemske 1985; Goodwillie et al. 2005; Charlesworth and Willis 2009). However, perhaps surprisingly, no equivalent theory explicitly considers the dynamics of alleles underlying inbreeding avoidance or inbreeding preference given obligate biparental reproduction or, hence, predicts the circumstances under which such alleles are likely to increase in frequency. 
Obligate biparental reproduction introduces multiple complex factors that affect inbreeding and might, consequently, affect allele frequency dynamics (Duthie and Reid 2015). First, selection on inbreeding is widely predicted to be sex specific (e.g., Parker 1979, 2006; Waser et al. 1986; Kokko and Ots 2006). The transmission advantage of alleles causing inbreeding is smaller in individuals of the resource-limited sex (typically females) because inbreeding cannot increase the individual's own reproductive success, only that of their related mates (Parker 1979, 2006; Waser et al. 1986). The advantage is greater in individuals of the mate-limited sex (typically males) because inbreeding can increase the individual's own reproductive success (Parker 1979, 2006; Waser et al. 1986). Sexual conflict over inbreeding is, therefore, predicted (Parker 1979, 2006; Waser et al. 1986; Kokko and Ots 2006; Pizzari and Gardner 2012; Szulkin et al. 2013). In general, the consequences of such sexual conflict can be diverse (Rowe et al. 1994; Arnqvist and Rowe 2005; Parker 2006), complicating prediction of underlying allele frequency dynamics.

Second, unlike the simple dichotomy of self-fertilization versus outcrossing, the degree to which obligately biparental organisms can avoid or prefer inbreeding through mate choice must depend on the relatedness between each individual and its potential mates. The distribution of relatedness will, in turn, depend on population size and viscosity (Queller 1994; Pizzari and Gardner 2012; Pizzari et al. 2015) as well as on stochastic and deterministic variation in fitness among individuals and families, which might itself depend partly on the pattern of inbreeding in previous generations (Puurtinen 2011; Young et al. 2012; Szulkin et al. 2013; Zimmer et al. 2014).

Third, while opportunities to actively avoid or prefer biparental inbreeding might be greatest in small or viscous populations where both relatives and nonrelatives regularly interact, these same circumstances might impede evolution of any adaptive reproductive strategy. Selection is inefficient in small populations because allele frequency changes caused by selection can be overwhelmed by stochastic sampling effects (i.e., genetic drift). This same principle also applies to local selection in viscous populations, where increased drift occurs within demes, or among locally interacting individuals, connected by gene flow (Turner 1982; Travis and Ezard 2006). This implies that in populations that are sufficiently small or viscous to create opportunities for inbreeding avoidance or preference, selection might be too weak to drive evolution of any such inbreeding strategy. In contrast, when populations are large and panmictic, drift will be less influential, but selection on inbreeding strategy might be weak because individuals can rarely inbreed through either active mate choice or random mating.

Fourth, like self-fertilization, biparental inbreeding increases homozygosity and exposes deleterious recessive al- leles to selection (Barrett and Charlesworth 1991; Charlesworth and Willis 2009). The frequency of deleterious recessive alleles might, therefore, be decreased by biparental inbreeding (i.e., purging; Barrett and Charlesworth 1991; Hedrick 1994; Crnokrak and Barrett 2002; Benesh et al. 2014). Evolution of inbreeding preference might, therefore, reduce the magnitude of inbreeding depression, in turn, reducing selection against inbreeding, potentially leading to coevolution between inbreeding depression and inbreeding preference (Lande and Schemske 1985; Benesh et al. 2014). Conversely, if purging caused by biparental inbreeding were minimal, evolution of inbreeding preference might instead increase expression of mutation load and lead to population extinction (Hedrick 1994; Frankham et al. 2001). While coevolutionary feedbacks between mutation load and inbreeding strategy have been emphasized in the context of self-fertilization versus outcrossing (Lande and Schemske 1985; Charlesworth et al. 1990; Latta and Ritland 1993; Porcher and Lande 2005, 2013), no models have explicitly considered such coevolution given obligate biparental reproduction (Kokko and Ots 2006; Puurtinen 2011; Szulkin et al. 2013).

Fifth, evolution of inbreeding avoidance or preference is likely to be inhibited by multiple components of negative selection (i.e., costs). One obvious cost that is integral to all hypotheses that consider evolution of inbreeding is reduced fitness of inbred offspring (i.e., inbreeding depression; Parker 1979, 2006; Lande and Schemske 1985; Waser et al. 1986; Lloyd 1992; Lehmann and Perrin 2003; Kokko and Ots 2006). Evolution of inbreeding is further inhibited by opportunity costs, where inbreeding with a relative reduces or precludes an individual's ability to reproduce with other relatives or nonrelatives, thereby reducing the transmission advantage of an underlying allele (Parker 1979, 2006; Waser et al. 1986; Lloyd 1992; Keller and Arcese 1998; Porcher and Lande 2005; Charlesworth 2006). Furthermore, active inbreeding avoidance or preference might incur a direct fitness cost in the form of reduced survival or reproductive success of individuals that express mate choice rather than mating randomly, for example, reflecting costs of mate searching (e.g., Lehmann and Perrin 2003; Kokko and Ots 2006; Jamieson et al. 2009) or kin discrimination (e.g., Waser et al. 1986) or costs of dispersing to mate elsewhere (e.g., Bengtsson 1978; Gandon 1999).

Finally, unlike self-fertilization versus outcrossing, biparental inbreeding avoidance or preference requires individuals to discriminate among nonself relatives and nonrelatives and, thereby, bias their reproduction with respect to relatively subtle variation in relatedness. Discrimination can stem from broad spatial cues, such as when inbreeding avoidance is accomplished by sex-specific dispersal (e.g., Pusey 1987; Perrin and Mazalov 2000; Lehmann and Perrin 2003; Lawson Handley and Perrin 2007). However, in less dispersive systems, direct discrimination among potential mates of different re- 
latedness might be required (e.g., Frommen and Bakker 2006; Langen et al. 2011; Lemaître et al. 2012; Herfindal and Haanes 2014). While individuals from diverse taxa can discriminate familiar or close relatives from unfamiliar relatives or nonrelatives (Griffin and West 2003; Dudley and File 2007; Strassmann et al. 2011), discrimination among distant relatives might be ineffective (but see Mateo 2002), potentially constraining evolution of biparental inbreeding strategy.

Overall, the complexities stemming from the combined effects of sexual conflict, distribution of relatedness, population size, purging, multiple costs, and kin discrimination make it unclear whether selection should be expected to cause an initially rare mutant allele underlying inbreeding avoidance or inbreeding preference to increase in frequency in a population with obligate biparental reproduction. Accordingly, we built an individual-based model to explicitly track the dynamics of mutant alleles causing inbreeding avoidance or preference in populations of different sizes that are initially fixed for inbreeding tolerance (i.e., random mating with respect to relatedness). We consider allele dynamics when females or males control mating interactions and, given different magnitudes of inbreeding depression, direct costs of nonrandom mating and restrictions on kin discrimination. We thereby determine the conditions under which selection will favor alleles causing active strategies of inbreeding avoidance or preference through mate choice given internally consistent feedbacks among relatedness structure, mutation load, and inbreeding strategy.

\section{Model}

We model reproductive interactions among females and males in a dioecious population with nonoverlapping generations and no explicit spatial structure. In each simulation, either females or males are designated as the choosing sex, and only individuals of the choosing sex can actively choose mate(s). This allows evolutionary consequences of any sexual conflict that might arise to be controlled by one sex or the other because selection on inbreeding strategy acts directly only on choosing sex individuals. A single generation proceeds with mate choice, reproduction, offspring mortality, immigration, and density regulation (fig. A1; figs. A1-A3, B1 available online; $\mathrm{C}$ code is deposited in the Dryad Digital Repository: http://dx.doi.org/10.5061/dryad.3861f [Duthie and Reid 2016]). ${ }^{1}$ We first describe the attributes of individuals (fig. A2), then describe how individuals interact, and finally summarize simulation specifications.

1. Code that appears in The American Naturalist is provided as a convenience to the readers. It has not necessarily been tested as part of the peer review.

\section{Individual Attributes}

Each individual has a diploid genome, comprising 1,002 physically unlinked loci. One locus affects inbreeding strategy and has three possible alleles. Wild-type alleles $\left(S^{+}\right)$cause inbreeding tolerance (i.e., random mating with respect to relatedness). Inbreeding-avoidance alleles $\left(S^{A}\right)$ decrease the perceived quality of related potential mates and, hence, lead to less inbreeding than expected given random mating. Inbreeding-preference alleles $\left(S^{P}\right)$ increase the perceived quality of related potential mates and, hence, lead to greater inbreeding than expected given random mating. To isolate the effects of selection on a mutant allele underlying inbreeding avoidance or preference within a single population, $S^{+}$ can mutate to either $S^{A}$ or $S^{P}$ but not to both. Therefore, inbreeding avoidance and preference cannot both evolve in the same population. A second locus has two neutral alleles, one wild type $\left(N^{+}\right)$and one mutant $\left(N^{-}\right)$, that have no effect on mate choice. Allele frequencies at the $N$ locus are, therefore, affected by drift and mutation but not directly by selection. Selection on $S^{A}$ or $S^{P}$ can, therefore, be inferred by comparing the frequencies of these alleles to the frequency of $N^{-}$. Finally, each individual has 1,000 diploid diallelic loci, each of which can a have wild-type allele $\left(L^{+}\right)$or a deleterious recessive mutant allele $\left(L^{-}\right)$. Expression of resulting mutation load decreases offspring survival probability, thereby causing inbreeding depression (following Guillaume and Perrin 2009).

\section{Model Overview}

At the start of each generation, individuals of the choosing sex that carry at least one allele underlying an active inbreeding strategy $\left(S^{A}\right.$ or $\left.S^{P}\right)$ incur a direct cost $(c)$, modeled as a probability of being unable to mate $c$ for heterozygotes (e.g., $S^{+} S^{A}$ ) and $2 c$ for homozygotes (e.g., $S^{A} S^{A}$ ). Because generations are nonoverlapping, individuals that are unavailable to mate have zero fitness, constituting a direct cost of alleles underlying nonrandom mating (Bengtsson 1978; Pomiankowski 1987; Pomiankowski et al. 1991).

Individuals of the choosing sex deemed available to mate are randomly ordered. Sequentially, each individual simultaneously examines all available members of the nonchoosing sex and assigns a perceived quality value to each potential mate based on $S$ alleles, kinship, and kin discrimination. Kinship between two individuals $i$ and $j\left(k_{i j}\right)$ is the probability that randomly sampled homologous alleles will be identical by descent (Lynch and Walsh 1998), calculated using a standard iterative algorithm incorporating parent-offspring relationships (Boyce 1983). The quality that a choosing individual $i$ assigns to a potential mate $j$ is a linear function of $k_{i j}$. If $i$ has a single $S^{A}$ allele, the perceived quality of $j$ is decreased by $\alpha \times k_{i j}$, where $\alpha$ is the effect size of a mutation affecting inbreeding strategy (sensu Verzijden et al. 2005; Proulx and 
Servedio 2009; Olofsson et al. 2011). Nonkin are assigned a default quality of 1 , so $i$ is $1+\alpha k_{i j}$ times less likely to choose to mate with $j$ than with an available nonrelative (i.e., $j$ 's perceived quality is $\left.1 /\left(1+\alpha k_{i j}\right)\right)$. Inbreeding strategy alleles have additive effects, so if $i$ is homozygous for $S^{A}, i$ is $1+$ $2 \alpha k_{i j}$ times less likely to choose to mate with $j$ than with an available nonrelative (i.e., $j$ 's perceived quality is $1 /(1+$ $\left.\left.2 \alpha k_{i j}\right)\right)$. Similarly, if $i$ has one or two $S^{P}$ alleles, they are $1+$ $\alpha k_{i j}$ and $1+2 \alpha k_{i j}$ times more likely to choose to mate with $j$ than with an available nonrelative, respectively. In primary simulations, the choosing individual $i$ is assumed to have perfect kin discrimination and, hence, exact knowledge of $k_{i j}$ for all potential mates. For subsequent simulations, kin discrimination is restricted such that only full and half siblings are recognized as relatives, and all other individuals are perceived as unrelated and assigned a quality of 1 . Mate choice is realized by sampling from a probability vector whose elements comprise the perceived qualities of all of an individual's available mates' qualities divided by the sum of these qualities.

To model stereotypical sex roles, females can mate once, and males can mate up to $\Omega$ times (i.e., limited polygyny). When females choose mates, there is some opportunity cost of male mating because mating with one female might prevent a male from mating with another female by using a unit of the male's quota of $\Omega$ (Waser et al. 1986). Male opportunity cost, therefore, decreases with increasing $\Omega$, because at high $\Omega$, a male's quota is less likely to be filled. In contrast, when males choose mates, each can choose $\Omega$ females, but each female can be chosen only once. This creates a strong but realistic (see "Discussion") opportunity cost when males choose because mating with one female always prevents a male from mating with another female by using up a unit of $\Omega$ (Waser et al. 1986). When males choose, each randomly ordered male chooses $\Omega$ females before the next male starts choosing. Once all females are chosen, remaining males cannot mate, generating variance in male mating success. Each female that mates produces $n$ offspring; females and males are produced with equal probability.

For all 1,002 loci, one allele from each parent is randomly inherited by each offspring. Each inherited allele mutates independently with probability $\mu$, with equal likelihood of mutation from wild type to mutant and mutant to wild type. An offspring's survival probability ( $\left.\Psi_{\text {off }}\right)$ depends on its total numbers of $L^{-} L^{-}$homozygous $\left(\Theta_{\text {off }}\right)$ and $L^{+} L^{-}$heterozygous $\left(\theta_{\text {off }}\right)$ genotypes, on the selection coefficient $(s)$ against $L^{-}$, and on $L^{- \text {s }}$ s dominance coefficient $(h)$, such that,

$$
\Psi_{\text {off }}=(1-s)^{\Theta_{\text {off }}} \times(1-h s)^{\theta_{\text {off }}} .
$$

Equation (1) assumes that independent loci have multiplicative genetic effects on survival probability (Morton et al. 1956; Mills and Smouse 1994). Inbreeding depression arises because inbreeding increases the probability that deleterious recessive alleles will be homozygous, increasing $\Theta_{\text {off }}$ and de- creasing $\Psi_{\text {off }}$ (if $h$ is zero or small; table 1). Survival is realized using an independent Bernoulli trial for each offspring. Within a single simulation, $s$ is uniform over all $L$ loci and generations (i.e., all deleterious recessive alleles have the same effect; Roze and Rousset 2005). Therefore, we model inbreeding depression as hard rather than soft selection (i.e. absolute rather than relative). Hard selection allows us to model the population dynamic consequences of inbreeding (e.g., extinction) and ensures that the realized impact of $s$ can be varied consistently over generations and simulations. If selection was soft, different $s$ values might not translate into different magnitudes of inbreeding depression given identical distributions of kinship and $S^{A}$ or $S^{P}$ frequencies.

After offspring mortality, all adults die, and then surviving offspring immediately become adults. Next, $\rho$ immigrants arrive and are added to the population. Immigrants are unrelated to all individuals within the native population and to other immigrants. Immigration is modeled only to prevent

Table 1: Definitions of model alleles, parameters, and variables

\begin{tabular}{|c|c|c|}
\hline & Description & Default \\
\hline \multicolumn{3}{|l|}{ Allele: } \\
\hline$S^{+}$ & Inbreeding tolerance & NA \\
\hline$S^{A}$ & Inbreeding avoidance & NA \\
\hline$S^{P}$ & Inbreeding preference & NA \\
\hline$N^{+}$ & Neutral wild type & NA \\
\hline$N^{-}$ & Neutral mutant & NA \\
\hline$L^{+}$ & Load wild type & NA \\
\hline$L^{-}$ & Load deleterious & NA \\
\hline \multicolumn{3}{|c|}{ Parameter: } \\
\hline$s$ & Selection coefficient against $L^{-}$allele & $0-.02$ \\
\hline$c$ & Direct cost of inbreeding strategy & $0-.01$ \\
\hline$h$ & $\begin{array}{l}\text { Dominance coefficient of deleterious } \\
\text { alleles }\end{array}$ & 0 \\
\hline$n$ & Offspring produced per female & 5 \\
\hline$\Omega$ & Maximum mates per male & 5 \\
\hline$\rho$ & Immigrants per generation & 5 \\
\hline$K_{\mathrm{f}}$ & Female carrying capacity & 100 \\
\hline$K_{\mathrm{m}}$ & Male carrying capacity & 100 \\
\hline$\mu$ & Allele mutation rate & .001 \\
\hline$\alpha$ & $\begin{array}{l}\text { Strength of inbreeding } \\
\text { avoidance/preference }\end{array}$ & 10 \\
\hline \multicolumn{3}{|c|}{ Variable: } \\
\hline$k$ & $\begin{array}{l}\text { Coefficient of kinship between } \\
\text { individuals }\end{array}$ & NA \\
\hline$f_{\text {off }}$ & Offspring coefficient of inbreeding & NA \\
\hline$\Psi_{\text {off }}$ & Offspring survival probability & NA \\
\hline$\Theta_{\text {off }}$ & $\begin{array}{l}\text { Offspring number of homozygous } \\
L^{-} L^{-} \text {loci }\end{array}$ & NA \\
\hline$\theta_{\text {off }}$ & $\begin{array}{l}\text { Offspring number of heterozygous } \\
L^{+} L^{-} \text {loci }\end{array}$ & NA \\
\hline$N_{\mathrm{e}}$ & Effective population size & NA \\
\hline
\end{tabular}

Note: $\mathrm{NA}=$ not applicable. 
the native population from becoming completely inbred, not to introduce a further cause of allele frequency change. Immigrants are always of the nonchoosing sex, thereby preventing immigration from directly affecting evolution of inbreeding strategy. If immigrants were of the choosing sex, any immigrant inbreeding-preference or inbreeding-avoidance alleles would not be visible to selection because immigrants are assumed to have no relatives with which to inbreed. Immigrants' alleles are independently drawn for the $S$ and $N$ loci, with probabilities equal to the allele frequencies of the native population at the time of immigration. The frequency of $L^{-}$ is averaged over all 1,000 loci of all native individuals, and this frequency is used to draw $L^{-}$versus $L^{+}$for each $L$ locus in each immigrant, thereby preventing the expected genetic load from changing due to immigration. Conceptually, we thereby model a small native population embedded within a larger, highly viscous population with demes that are connected by occasional dispersers and where evolution of inbreeding strategy is assumed to be progressing uniformly across all demes.

Finally, we set a carrying capacity of females $\left(K_{\mathrm{f}}\right)$ and males $\left(K_{\mathrm{m}}\right)$, which limits population size (e.g., Guillaume and Perrin 2009). Following immigration, if the number of females or males exceeds $K_{\mathrm{f}}$ or $K_{\mathrm{m}}$, respectively, random mortality reduces female and male population size to $K_{\mathrm{f}}$ and $K_{\mathrm{m}}$. Because density regulation follows immigration, female and male population sizes (and, therefore, the number of available mates in the next generation) never exceed $K_{\mathrm{f}}$ and $K_{\mathrm{m}}$, regardless of which sex chooses mates. Therefore, the degree to which outbred versus inbred matings occur does not inherently depend on which sex chooses. Surviving individuals enter the mating pool for the next generation of mate choice and reproduction. Parent and offspring identities are recorded to allow calculation of internally consistent pairwise $k_{i j}$ resulting from reproduction enacted across consecutive generations.

\section{Model Initialization and Primary Simulations}

We initialized all simulations with 100 founders of each sex, and $K_{\mathrm{f}}=K_{\mathrm{m}}=100$ for primary simulations. Simulations start with only wild-type alleles $S^{+}, N^{+}$, and $L^{+}$at all loci (i.e., no genetic variation); 100 generations pass with no mutation to burn in a kinship structure prior to evolution. After 100 generations, the probability that an allele mutates is $\mu=0.001$ (e.g., Guillaume and Perrin 2009), regardless of whether the allele is wild type (e.g., $L^{+}$) or mutant (e.g., $L^{-}$). For primary simulations, we set the strength of inbreedingavoidance and inbreeding-preference alleles to $\alpha=10$, the maximum number of mates per male to $\Omega=5$, and the number of immigrants per generation to $\rho=5$ (table 1 ). Separate simulations consider kin discrimination that is perfect versus restricted to full and half siblings.
We ran exploratory simulations to examine system dynamics and identify appropriate metrics of evolution (fig. B1). Frequencies of $S^{A}$ and $S^{P}$ varied, but we could not assume a priori that long-term dynamics of $S^{A}$ and $S^{P}$ would necessarily lead to extinction or fixation (e.g., if selection led to intermediate frequencies). Thus, rather than estimating allele fixation times or probabilities, we calculated the frequency of each allele after a fixed number of generations (hereafter referred to as end frequency).

Wild-type and mutant alleles are equally likely to mutate, so the population-wide frequency of forward (e.g., $N^{+} \rightarrow N^{-}$) and back (e.g., $N^{-} \rightarrow N^{+}$) mutations depends on how many wild-type and mutant alleles are in the population. This causes the long-term extinction and fixation of $N^{-}$to be equally likely after 3,000 generations, resulting in a Ushaped distribution of $N^{-}$end frequencies with a mean of 0.5 across replicate simulations (fig. A3; Mettler et al. 1969). To test whether long-term dynamics of $S^{A}$ or $S^{P}$ differ from $N^{-}$, we therefore compared $S^{A}$ and $S^{P}$ end frequency distributions after 3,000 generations to those of $N^{-}$across replicate simulations. Where mean end frequencies exceeded those of $N^{-}(>0.5)$, we inferred selection for $S^{A}$ or $S^{P}$. Where mean end frequencies were lower $(<0.5)$, we inferred selection against $S^{A}$ or $S^{P}$. Frequencies of $L^{-}$alleles varied due to drift, but mean $L^{-}$frequency summed across $L$ loci usually stabilized after 1,000 generations, as did the distribution of kinship (fig. B1).

We simulated across a range of selection coefficients $s=\{0.000,0.004, \ldots, 0.016,0.020\}$ and direct costs $c=$ $\{0.0000,0.0025,0.0050,0.0100\}$. Each combination of $s$ and $c$ was run for 100 replicate simulations under four sets of conditions: (1) female mate choice and a mutant $S^{A}$ allele, (2) male mate choice and a mutant $S^{A}$ allele, (3) female mate choice and mutant $S^{P}$ allele, and (4) male mate choice and a mutant $S^{P}$ allele. For each set of 100 replicates, we bootstrapped $95 \%$ confidence intervals around mean end frequencies of $S^{A}$ and $S^{P}$ (Manly 2007, p. 46). These confidence intervals are intended to help interpret patterns of allele end frequencies and are not tests of statistical significance (White et al. 2014).

\section{Effects of Effective Population Size}

To systematically evaluate the effect of genetic drift on the end frequency of $S^{A}$ and $S^{P}$ when females and males chose, we simulated across a range of carrying capacities $\left(K_{\mathrm{f}}=K_{\mathrm{m}}=\left\{6^{2}, 7^{2}, \ldots, 20^{2}, 21^{2}\right\} ; 100\right.$ replicates each) to generate different effective population sizes $\left(N_{\mathrm{e}}\right)$. The frequency of $N^{-}(q)$ was calculated in the last 1,000 generations of each simulation and used to calculate the expected frequency of $N^{-}(E[q])$ and the expected change in frequency of $N^{-}$in a generation $(E[\Delta q])$. For each replicate, 
we then calculated $N_{\mathrm{e}}$,

$$
N_{\mathrm{e}}=\frac{(E[q])(1-E[q])}{2(E[\Delta q])}
$$

(Wright 1931; Caballero 1994). Mean estimates of $N_{\mathrm{e}}$ and either $S^{A}$ or $S^{P}$ end frequencies were calculated over 100 replicate simulations. For these simulations, we used default parameter values and set $s=0.012$ and $c=0.0025$. To determine how $N_{\mathrm{e}}$ varied across other values of $s$ and $c$, we additionally estimated $N_{\mathrm{e}}$ using 10 replicates for all default parameter value combinations (supplemental PDF, pp. S6-S7).

\section{Nonchoosing Sex Affecting Quality}

In our primary simulations, mating is controlled entirely by the choosing sex because individuals of the nonchoosing sex have no effect on the probability that they will be chosen as mates. If both sexes influence mate choice, then frequencies of $S^{A}$ and $S^{P}$ might be intermediate to those observed when either sex has complete control. Alternatively, more complex interactions in which both sexes affect mating outcomes might drive the dynamics of allele frequencies in counterintuitive ways. Female versus male control cannot then be interpreted as representing opposite extremes. To explore one way that mate choice might be influenced by both sexes, we allow the inbreeding strategy alleles of nonchoosing sex individuals to affect how their quality is perceived by choosing sex individuals. Here alleles underlying inbreeding strategy in individuals of both sexes have combined additive effects on the perceived quality of potential mates. For example, in simulations with mutant $S^{P}$, the quality of a potential mate $j$ for individual $i$ of the choosing sex is increased by $\alpha k_{i j}$ for each $S^{P}$ allele in either individual. If $i$ is $S^{P} S^{P}$ and $j$ is $S^{P} S^{+}$, then $i$ will be $1+3 \alpha k_{i j}$ times more likely to mate with $j$ than an available nonrelative. Similarly, in simulations with $S^{A}$, an $S^{A} S^{A}$ individual $i$ will be $1+3 \alpha k_{i j}$ times less likely to mate with an $S^{A} S^{+}$individual $j$ than an available nonrelative. Simulations in which nonchoosing sex individuals affected their own perceived quality were run using default parameter values (table 1).

\section{Inbreeding Depression and Purging}

To link $s$ values to inbreeding depression in offspring survival as might be estimated in wild populations, we regressed the natural logarithm of offspring survival probability $\Psi_{\text {off }}$ on offspring inbreeding coefficient $f_{\text {off }}$, such that $E\left[\ln \left(\Psi_{\text {off }}\right)\right]=\beta_{0}+\beta_{1} f_{\text {off }}$. The coefficient $\beta_{0}$ estimates load that is independent of inbreeding, and $\beta_{1}$ estimates load attributable to inbreeding expressed in terms of haploid lethal equivalents (Morton et al. 1956). The value of $f_{\text {off }}$ for any offspring of parents $i$ and $j$ equals $k_{i j}$ (Lynch and Walsh
1998). Because inbreeding depression is typically reported as diploid lethal equivalents (Ralls et al. 1988; O'Grady et al. 2006), we present inbreeding depression measured as $2 \beta_{1}$ rather than $\beta_{1}$.

To test for purging of deleterious recessive alleles and, hence, decreasing population-wide load, we recorded mean end frequency of $L^{-}$across all $L$ loci and $S^{A}$ and $S^{P}$ frequencies after 3,000 generations for all simulated magnitudes of $s$ when $c=0$. We regressed the frequency of $L^{-}$against the frequencies of $S^{A}$ and $S^{P}$ among replicates. If populations in which inbreeding preference is common tend to purge deleterious recessive alleles more than populations where inbreeding preference is rare, end frequencies of $L^{-}$should be negatively correlated with those of $S^{P}$.

\section{Sensitivity Analysis}

For $h, n, \Omega, \rho, K, \mu$, and $\alpha$ (table 1 ), we simulated 100 replicates for four nondefault values to determine sensitivities of end frequencies of $S^{A}$ and $S^{P}$. We restricted selection coefficient values to $s=\{0.000,0.004, \ldots, 0.016,0.020\}$ and direct costs to $c=0$. End frequencies of $S^{A}$ and $S^{P}$ were not qualitatively affected by $h, n, \Omega, \rho$, or $\mu$ (supplemental PDF, pp. S13-S22) but were affected by $K$ and $\alpha$ (see “Results").

\section{Results \\ Inbreeding Avoidance: Females Choosing Sex}

Figure $1 A$ summarizes the distributions of end frequencies of $S^{A}$ when females were the choosing sex. Each box summarizes 100 replicate simulations for different selection coefficients (rows) and direct costs (columns). Red horizontal lines and shading show the mean end frequencies and $95 \%$ bootstrapped confidence intervals. For comparison, blue circles in figure $1 \mathrm{~A}$ show the distribution of end frequencies of $N^{-}$for $c=0$. Mean $N^{-}$end frequency did not differ from 0.5 for different values of $s$ or $c$, so mean end $S^{A}$ frequency can be directly compared with end $E[q]=0.5$ (blue horizontal lines). Where distributions of $S^{A}$ end frequencies do not differ from those of $N^{-}$and confidence intervals overlap 0.5 , selection on $S^{A}$ is inferred to be weak.

Figure $1 A$ shows that the end frequency of $S^{A}$ varied greatly among replicate simulations when females chose and that $S^{A}$ was not consistently at or near fixation or extinction. For many parameter combinations, $S^{A}$ end frequency distributions were similar to $N^{-}$, implying that the considerable variation observed in $S^{A}$ end frequency largely reflected drift. Nevertheless, some patterns of structured variation were also evident. When $c=0$ and $s>0, S^{A}$ always reached a higher mean end frequency than $N^{-}$, and the mean end frequency increased with $s$ (rows top to bottom). 


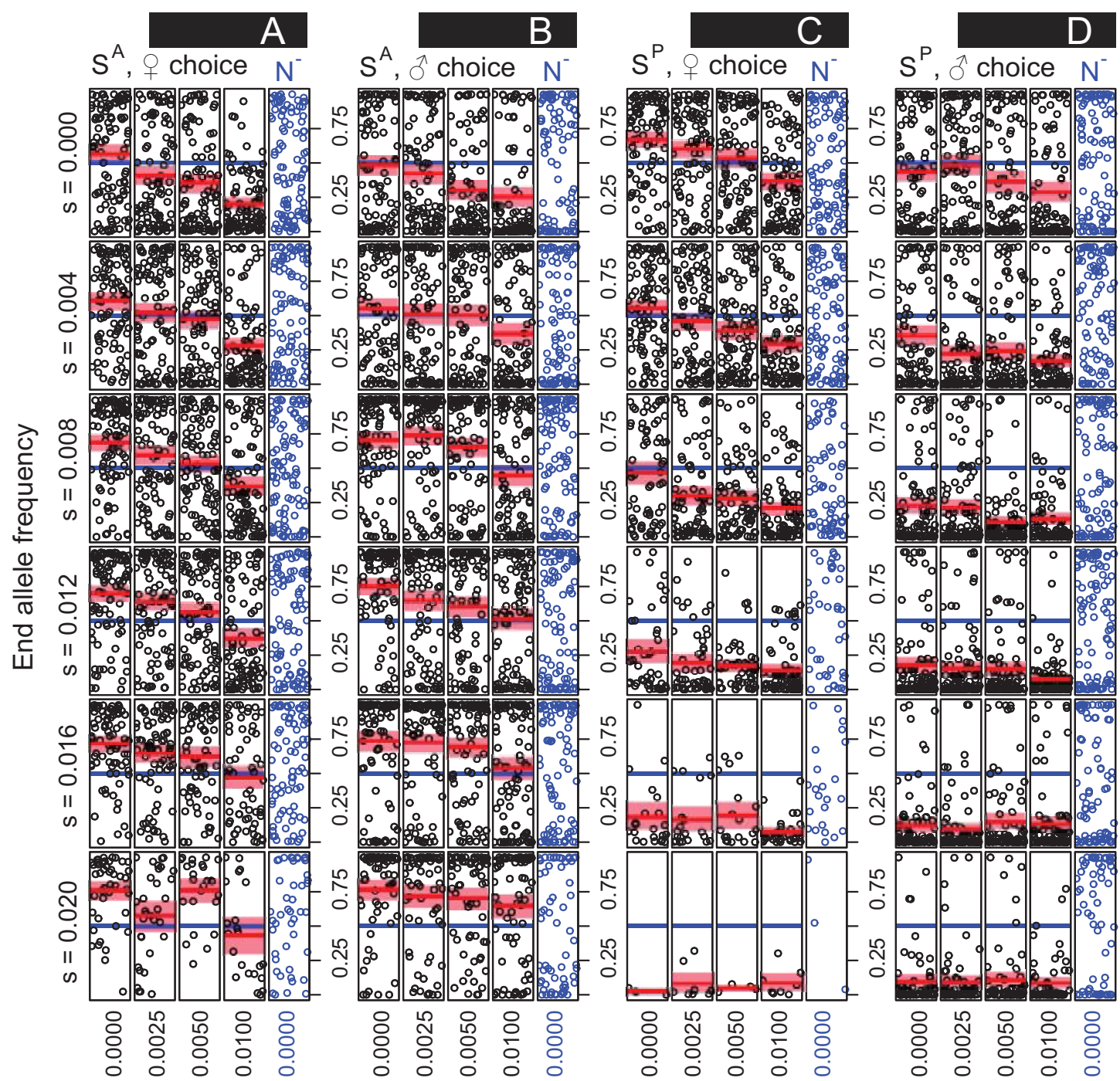

Cost of mate choice (c)

Figure 1: Distributions of end frequencies of alleles underlying inbreeding avoidance $\left(S^{A}\right)$ and inbreeding preference $\left(S^{P}\right)$ after 3,000 generations. The large columns present results with a mutant $S^{A}$ allele when females $(A)$ and males $(B)$ choose mates and of a mutant $S^{P}$ when females $(C)$ and males $(D)$ choose mates. Within each large column, the four left-hand columns of boxes present results with different direct costs $(c)$ of mate choice. Rows of boxes present results with different selection coefficients $(s)$ against deleterious recessive alleles. Each box presents 100 replicate simulations for a unique set of parameter combinations, and circles within boxes show mean end allele frequencies for a single simulation. Boxes with fewer than 100 circles reflect extinction. Along the $Y$-axis, circles show end allele frequencies from 0 to 1. Circles are randomized on the $X$-axis to facilitate visualization; the $X$-axis, therefore, does not convey information. Solid horizontal red lines show mean end allele frequencies, and red shaded regions show the $95 \%$ bootstrapped confidence intervals around the means. Solid blue lines show expected end frequencies under mutation-drift balance (0.5). For comparison, blue circles in the right-hand boxes of $A-D$ show the distribution of mutant neutral allele $\left(N^{-}\right)$end frequencies across replicates where $c=0.005$.

At high values of $s$, populations often went extinct (supplemental PDF, p. S3). As $c$ increased, the $S^{A}$ end frequency decreased (fig. 1A). Selection for inbreeding avoidance, therefore, occurred, especially given moderate to strong inbreeding depression and low direct costs, but $S^{A}$ did not always go to fixation under any simulated conditions.
Interestingly, when there was no inbreeding depression or direct cost (fig. $1 A ; s=0 ; c=0$ ), the $S^{A}$ end frequency was often $>0.5$ (one-tailed $t$-test; $P=.08$ ), and mean $S^{A}$ end frequency increased with increasing $\alpha$ (supplemental PDF, p. S19). Mean $S^{A}$ end frequency was, therefore, higher than expected under drift-mutation balance even in the ab- 
sence of inbreeding depression given a sufficiently strong effect of an $S^{A}$ allele. This was caused by runaway evolution driven by a positive correlation between female inbreeding avoidance and sons' reproductive success (see supplemental PDF p. S20).

\section{Inbreeding Avoidance: Males Choosing Sex}

Figure $1 B$ shows distributions of $S^{A}$ end frequencies when males were the choosing sex. Mean $S^{A}$ end frequency increased with increasing $s$ (rows top to bottom) and decreased with increasing $c$ (columns left to right), leading to mean end frequencies exceeding 0.5 for many parameter combinations. At high $s, S^{A}$ frequencies often ended at or near fixation.

In general, mean $S^{A}$ end frequencies had similar values whether females or males chose given identical $s$ and $c$ combinations (compare fig. $1 \mathrm{~A}, 1 \mathrm{~B}$ ). But population extinction was less likely when males chose mates (supplemental PDF, p. S3). This was because the native population received female immigrants when males chose, thereby increasing population growth through the production of outbred offspring of female immigrants.

\section{Inbreeding Preference: Females Choosing Sex}

Figure $1 C$ shows distributions of $S^{P}$ end frequencies when females were the choosing sex. When $c=0$ and $s=0$, mean $S^{P}$ end frequency was higher than that of $N^{-}$. Increasing $s$ caused a monotonic decrease in mean $S^{P}$ end frequency. High $s$ resulted in extinction of nearly all populations, with $97 \%$ of replicates going extinct when $s=0.02$ (supplemental PDF, p. S3). Overall, although selection for inbreeding preference sometimes occurred when females chose mates, the mean end frequency of $S^{P}$ exceeded that expected due to drift given only very small $s$ and $c$.

\section{Inbreeding Preference: Males Choosing Sex}

Figure $1 D$ shows distributions of $S^{P}$ end frequencies when males were the choosing sex. Mean $S^{P}$ end frequency never exceeded that of $N^{-}$and, therefore, never exceeded that expected due to drift. As in simulations with a mutant $S^{A}$ where males chose, populations rarely went extinct (supplemental PDF, p. S3).

\section{Effects of Effective Population Size}

Values of $N_{\mathrm{e}}$ as estimated from primary simulations were higher when females rather than males chose mates (supplemental PDF pp., S6-S7). This is because when males chose, $N_{\mathrm{e}}$ decreased due to increased variance in male reproductive success. Lower $N_{\mathrm{e}}$ when males chose can also be inferred from the higher proportion of extreme $N^{-}$end frequencies (indicating increased drift; fig. $1 B, 1 D$ ) compared to where females chose (fig. $1 A, 1 C$ ). Values of $N_{\mathrm{e}}$ also decreased slightly with increasing $s$ due to reduced census population size caused by inbreeding depression, but $N_{\mathrm{e}}$ was not affected by $c$ (supplemental PDF, pp. S6-S7).

Figure 2 shows how the end frequencies of mutant $S^{A}$ and $S^{P}$ alleles varied when values of $N_{\mathrm{e}}$ were imposed a priori by increasing population carrying capacity and, thereby, disentangles selection from drift because $N_{\mathrm{e}}$ defines the magnitude of drift. End frequencies of $S^{A}$ decreased as $N_{\mathrm{e}}$ increased when either females or males chose. This is because random mating was less likely to result in close inbreeding, decreasing selection for $S^{A}$. Conversely, end frequencies of $S^{P}$ did not change with $N_{\mathrm{e}}$ when females chose (fig. $2 A$ ) but increased slightly with increasing $N_{\mathrm{e}}$ when males chose (fig. $2 B$ ). The latter increase might have been caused by decreased selection against $S^{P}$ with increasing $N_{\mathrm{e}}$, as males carrying $S^{P}$ became less likely to successfully inbreed and, hence, experience negative selection. However, population extinction restricted estimation of allele end frequencies when females chose, and extinction was relatively common in simulations with mutant $S^{P}$ (supplemental PDF, p. S3). Overall, although drift is, by definition, greater at lower $N_{\mathrm{e}}$, selection for $S^{A}$ or against $S_{P}$ was not greatly weakened. Effects of $N_{\mathrm{e}}$ on $f_{\text {off }}$ and $L^{-}$frequency were negligible (supplemental PDF, pp. S8-S9).

\section{Synthesis of Inbreeding Depression, Kinship, and Purging}

Figure 3 synthesizes inbreeding strategy allele end frequencies in relation to $2 \beta_{1}$ across choosing sexes and direct costs (c). The mean end frequency of $S^{A}$ generally increased with increasing $2 \beta_{1}$ when either females or males chose (fig. $3 A$, $3 B$ ), while the mean end frequency of $S^{P}$ generally decreased with increasing $2 \beta_{1}$ (fig. $3 C, 3 D$ ). Mean end frequencies of $S^{A}$ and $S^{P}$ both decreased with increasing $c$. As expected, increasing $s$ increased inbreeding depression in offspring survival (supplemental PDF, p. S4). However, when males rather than females chose, $N_{\mathrm{e}}$ was relatively small (supplemental PDF, pp. S6-S7), and population-wide mean kinship was, therefore, relatively high (fig. 4; Wright 1931). This relatively high mean kinship caused increased homozygosity due to identity by descent and, therefore, caused an increased expression of load attributable to inbreeding depression $\left(\beta_{1}\right)$. Consequently, simulations with identical $s$ values produced higher $2 \beta_{1}$ values when males rather than females chose (shown by the greater separation between points on the $X$-axis in fig. $3 B, 3 D$ vs. $3 A, 3 C$ ).

Mean kinship between all pairwise combinations of population members decreased slightly with increasing $S^{A}$ frequency. When females chose, mean kinship was highly sensitive to $S^{P}$; kinship increased with increasing $S^{P}$ frequency (supplemental PDF, p. S23), causing high among-replicate 


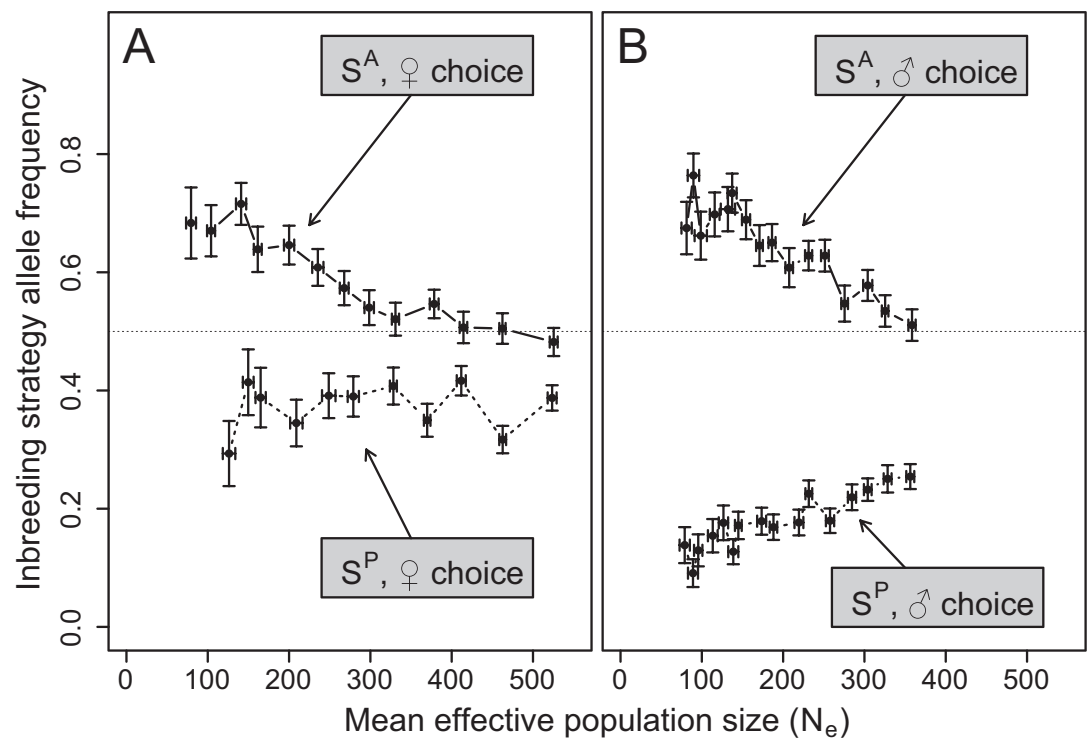

Figure 2: End frequencies of mutant alleles underlying inbreeding avoidance $\left(S^{A}\right)$ and preference $\left(S^{P}\right)$ after 3,000 generations across replicate simulations of an individual-based model with different mean effective population sizes $\left(N_{\mathrm{e}}\right)$. Four lines connecting points illustrate simulations of a mutant $S^{A}$ (solid) or $S^{P}$ (dotted) allele when either females $(A)$ or males $(B)$ choose mates. Each point represents a mean over 100 replicate simulations with identical starting conditions, and error bars show standard errors.

variation in end mean kinship values (fig. 4B). For other parameter combinations, mean kinship varied little among replicates (fig. $4 A, 4 C, 4 D$ ).

The effect that $S^{A}$ and $S^{P}$ had on mean kinship did not result in a change in mean end frequencies of $L^{-}$. Further, mean $L^{-}$frequencies were not affected by the choosing sex or $c$ but did decrease with increasing $s$ (fig. 4). For all $s$ values, the frequency of $L^{-}$did not strongly increase with the frequency of $S^{A}$ (regression slopes ranged from -0.001 to 0.003 ) or decrease with the frequency of $S^{P}$ (slopes ranged from -0.018 to 0.005 ; see supplemental PDF, p. S5). Purging, therefore, did not substantially affect $L^{-}$frequencies or, hence, mutation load.

\section{Restricted Kin Discrimination}

The dashed lines in figure 3 summarize how mean end frequencies of $S^{A}$ and $S^{P}$ alleles varied in relation to $2 \beta_{1}$ when kin discrimination was restricted to full or half siblings (full frequency distributions are shown in supplemental PDF, p. S11). Overall, mean $S^{A}$ end frequency decreased when kin discrimination was restricted, and mean $S^{A}$ end frequency never exceeded 0.5 when females chose (fig. $3 A$ ). When males chose, mean $S^{A}$ end frequency was higher than 0.5 only if $c=$ 0 and given sufficiently high $2 \beta_{1}$ (fig. $3 B$ ). Evolution of inbreeding avoidance was, therefore, inhibited when choosing individuals were only able to recognize full and half siblings relative to otherwise analogous conditions with perfect kin discrimination.
In contrast, restricted kin discrimination affected mean $S^{P}$ end frequency differently depending on the choosing sex. When females chose, mean $S^{P}$ end frequency was higher for identical $s$ and $c$ values with restricted discrimination than with perfect discrimination (fig. $3 C$ ). But when males chose, mean $S^{P}$ end frequency was always low irrespective of $c$ and $2 \beta_{1}$ (fig. 3D). Evolution of inbreeding preference was, therefore, facilitated by restricted kin discrimination when females chose but inhibited when males chose mates.

\section{Nonchoosing Sex Affecting Quality}

The dotted lines in figure 3 summarize how mean end frequencies of $S^{A}$ and $S^{P}$ varied in relation to $2 \beta_{1}$ when alleles carried by nonchoosing sex individuals influenced how their quality was perceived by individuals of the choosing sex (full frequency distributions are shown in supplemental PDF, p. S12). When females chose and males influenced their own quality, $S^{A}$ always ended at or near extinction (fig. $3 A$ ), and $S^{P}$ always ended at or near fixation (leading to population extinction when $s>0.008$; fig. $3 C$ ).

In contrast, when males chose, mean end frequencies of $S^{A}$ and $S^{P}$ were not strongly affected by whether females influenced how their quality was perceived (fig. $3 B, 3 D$ ). Where differences did occur, $S^{A}$ and $S^{P}$ end frequencies tended to be slightly higher when females influenced their perceived quality than when they did not (dotted vs. solid lines in fig. $3 B$, $3 D$ ). Overall, allowing individuals of the nonchoosing sex to affect how their own quality was perceived, therefore, had a 


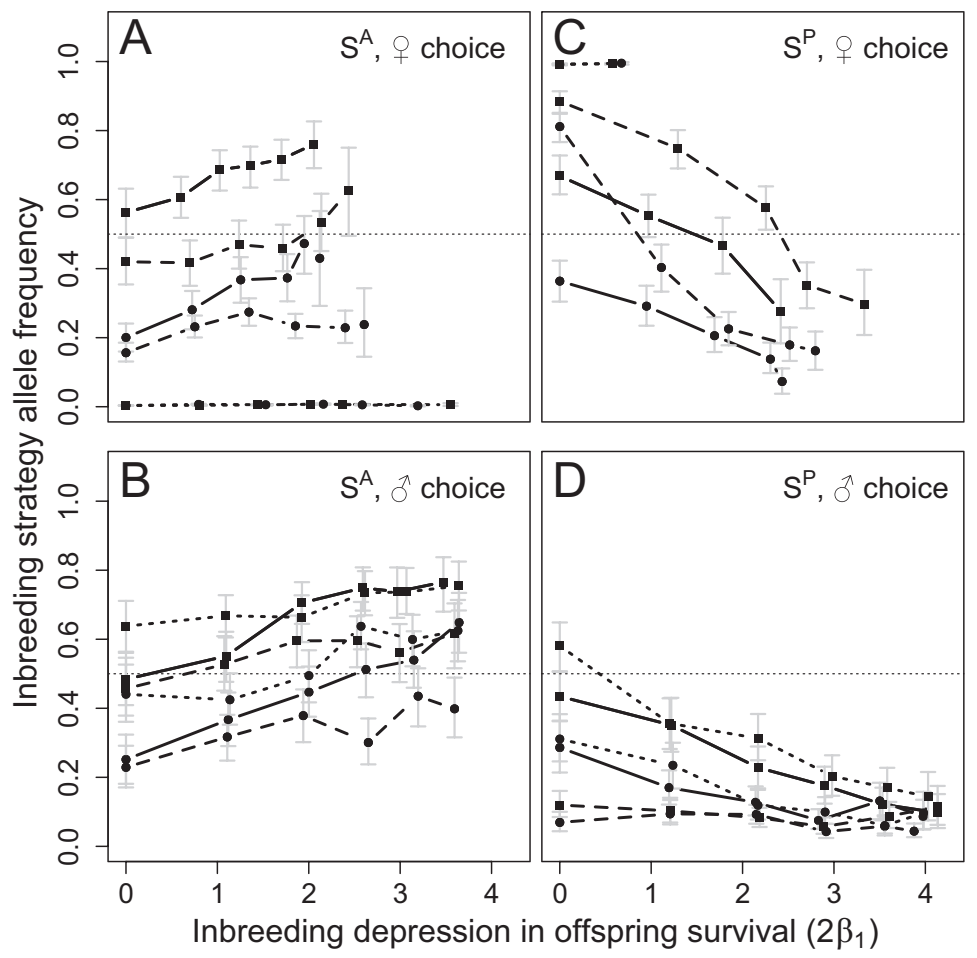

Figure 3: Summary of mean end frequencies of an inbreeding-avoidance allele $S^{A}$ when females $(A)$ and males $(B)$ choose mates and of an inbreeding-preference allele $S^{P}$ when females $(C)$ and males $(D)$ choose mates across 100 replicate simulations for different mean magnitudes of inbreeding depression $\left(2 \beta_{1}\right)$. Lines connect points across different selection coefficients $(s)$ underlying $2 \beta_{1}$ for combinations of cost $(c)$, kin discrimination, and nonchoosing sex effect on mate choice. Points show simulations in which the cost $(c)$ of inbreeding avoidance or preference is 0.00 (squares) and 0.01 (circles). Solid lines show primary simulations in which kin discrimination is perfect and the nonchoosing sex does not affect its perceived quality. Dashed lines show simulations in which kin discrimination is restricted and the nonchoosing sex does not affect its perceived quality. Dotted lines show simulations in which kin discrimination is perfect and the nonchoosing sex does affect its perceived quality. Error bars show 95\% bootstrapped confidence intervals (CIs) around the mean end frequencies; 95\% CIs around inbreeding depression are negligible (always $<0.01$ ) and are not shown. Thin dotted horizontal lines demarcate expected end frequencies under mutation-drift balance. Parameter combinations where $>50 \%$ of replicate populations went extinct are not shown.

large effect on $S^{A}$ and $S^{P}$ end frequencies when females, but not males, chose mates.

\section{Discussion}

Biparental inbreeding often decreases offspring fitness, creating widespread presumptions that inbreeding avoidance will inevitably evolve (Perrin and Mazalov 1999; Keller and Waller 2002; Geffen et al. 2011; Szulkin et al. 2013; Wright et al. 2013; Tennenhouse 2014). However, inbreeding avoidance might impose costs, while inbreeding can increase an individual's inclusive fitness, potentially driving evolution of inbreeding preference (Fisher 1941; Parker 1979, 2006; Szulkin et al. 2013; Porcher and Lande 2013). Indeed, recent studies report diverse evidence of inbreeding avoidance (e.g., Brouwer et al. 2011; Kingma et al. 2013; Banks and Lindenmayer 2013; Herfindal and Haanes 2014), preference (e.g., Wang and Lu 2011; Thünken et al. 2013), and tolerance (e.g., Olson et al. 2012; Nichols et al. 2014; Reynolds et al. 2014) in wild populations of biparentally reproducing species. However, no existing theory examines the dynamics of alleles underlying inbreeding avoidance or preference enacted through mate choice given key biological constraints affecting obligate biparental reproduction in small or viscous populations. Accordingly, we tested whether a mutant allele causing inbreeding avoidance or inbreeding preference increased in frequency in populations of potentially related females and males initially fixed for alleles causing inbreeding tolerance when females or males had control of mating and given explicit costs, kinship structure, mutation load, kin discrimination, and effective population size. Overall, selection for alleles underlying inbreeding avoidance occurred given sufficiently high inbreeding depression and low direct costs when both females and males chose, while selection for alleles underlying inbreeding preference occurred only when females chose and given very low inbreeding de- 


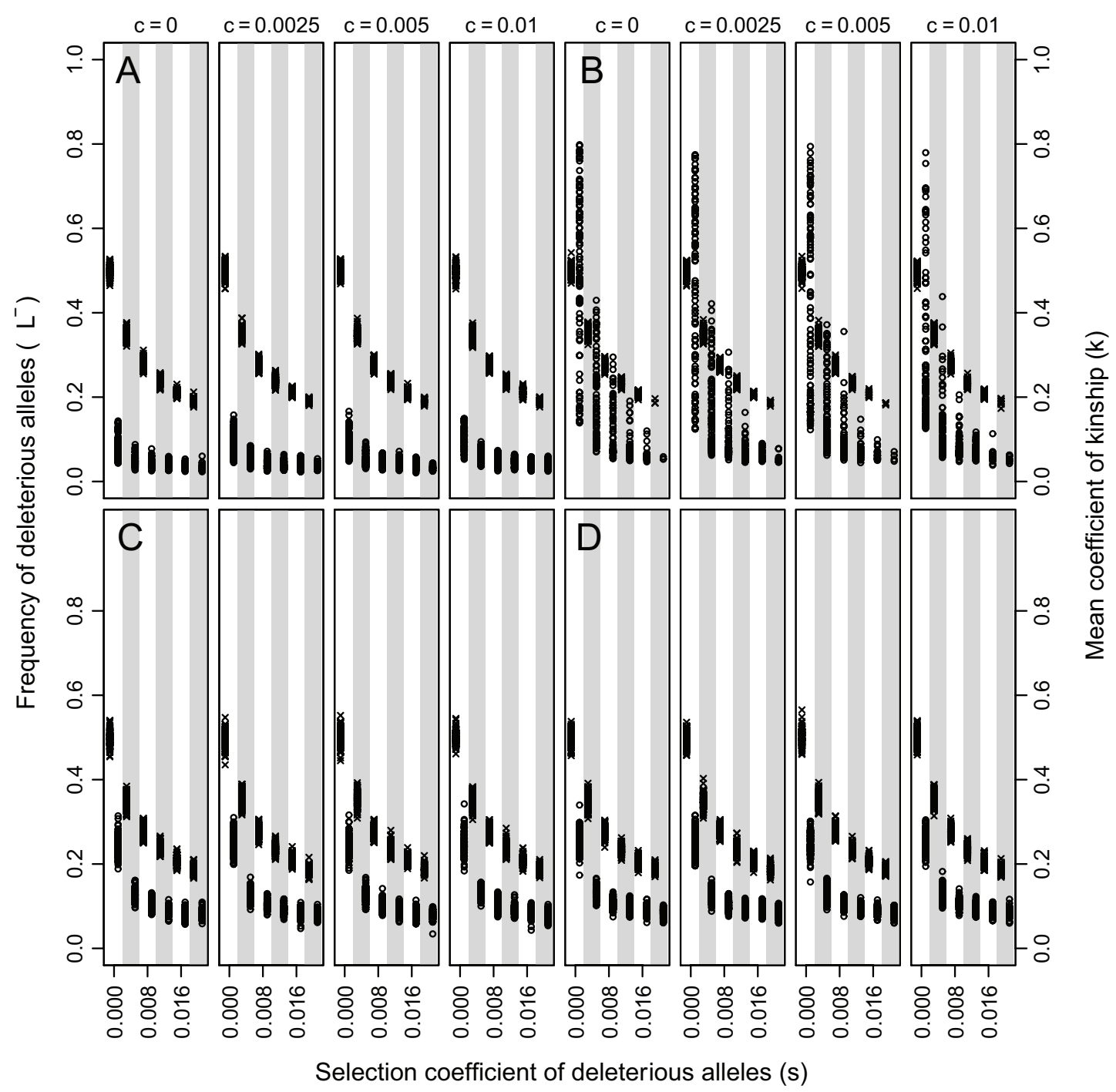

Figure 4: Distributions of the frequency of deleterious recessive $\left(L^{-}\right)$alleles (crosses; left side of lanes) and mean kinship (circles; right side of lanes) across replicate simulations where populations persisted for 3,000 generations, given an inbreeding-avoidance allele $\left(S^{A}\right)$ when females $(A)$ and males $(C)$ choose mates and an inbreeding-preference allele $\left(S^{P}\right)$ when females $(B)$ and males $(D)$ choose mates. Each panel shows distributions for four different costs $(c)$ of inbreeding strategy, and vertical lanes delineate different selection coefficients $(s)$ against deleterious alleles.

pression and direct costs. Selection for inbreeding avoidance weakened as census population size increased because random mating was less likely to result in inbreeding, but even in small populations, fixation of alleles underlying inbreeding avoidance was not inevitable due to genetic drift. Conditions favoring adaptive evolution of biparental inbreeding strategies may, therefore, be much more restricted than is often assumed.

\section{Population Size and Genetic Drift}

Our model illustrates that adaptive evolution of biparental inbreeding strategy is most likely in small and viscous pop- ulations. It is in these populations that relatives regularly interact, creating opportunities for selection on inbreeding avoidance or preference. Yet the effective size of these populations is also necessarily small at the local scale where mate choice occurs, meaning that dynamics of alleles affecting active inbreeding strategies will be strongly affected by drift (Mettler et al. 1969; Turner 1982; Travis and Ezard 2006), even when inbreeding strategies are adaptive. Nevertheless, studies that report an absence of inbreeding avoidance in wild populations rarely invoke genetic drift as a potential explanation (e.g., Keane et al. 1996; Keller and Arcese 1998; Hansson et al. 2006; Jamieson et al. 2009). Conversely, in large panmictic populations where drift will be weaker, 
the risk of inbreeding given random mating decreases, decreasing selection for alleles underlying inbreeding avoidance even given moderate inbreeding depression and precluding adaptive evolution of such strategies (fig. 2).

\section{Choosing Sex}

Evolution of inbreeding avoidance did not depend qualitatively on whether females or males were the choosing sex. The $S^{A}$ allele underlying inbreeding avoidance was always more likely to reach high frequencies when inbreeding depression was strong and direct costs of mate choice were low (fig. 3). In contrast, the $S^{P}$ allele underlying inbreeding preference was expected to reach high frequencies only when females, but not males, were the choosing sex and when both inbreeding depression and direct costs were very weak. This sex dependence arises because the number of offspring females can produce is limited to $n$. Consequently, a male cannot increase the reproductive success of his female relatives by preferentially mating with them. Male mating should simply be maximized, except when inbreeding depression causes selection for inbreeding avoidance (Pizzari et al. 2004). Males might prefer to mate with relatives rather than not mating but should never prefer to mate with relatives rather than nonrelatives (Puurtinen 2011), constraining selection for $S^{P}$. In contrast, because variation exists in male mating success and, hence, reproductive success (given $\Omega>1$ ), females can increase the reproductive success of their male relatives by preferentially mating with them. Hence, selection for inbreeding preference is predicted only under highly restricted conditions when females control mating.

Available empirical evidence broadly matches this prediction. For example, female great frigatebirds (Fregata minor) conduct mate choice (Nelson 1975) and are more closely related to their mates than expected given random mating (Cohen and Dearborn 2004). While social pairing occurs randomly with respect to relatedness in barn swallows (Hirundo rustica erythrogaster; Kleven et al. 2005) and ground tits ( $\mathrm{Pa}$ rus humilis; Wang and Lu 2011), females of both species apparently prefer related extra-pair mates. Furthermore, yellowbellied marmot (Marmota flaviventris) pairs are more closely related than expected given random mating (Olson et al. 2012). Marmots form social matrilines, and female reproduction is highly resource limited (Armitage 1998, 2003). Future empirical studies of inbreeding in such systems could usefully quantify mate choice behavior and sexual conflict using both observational and experimental approaches, as previously achieved in the cichlid fish Pelvicachromis taeniatus. Experiments showed that males use olfactory cues (Thünken et al. 2013) to distinguish related and unrelated females and actively choose related females as mates (Thünken et al. 2007a, $2007 b$, 2013). Observations of wild populations also suggest active inbreeding preference (Langen et al. 2011). Such maledriven inbreeding preference contradicts our prediction that inbreeding preference should not arise when males control mating. However, $P$. taeniatus might be an interesting case of sex role reversal. Teleost fishes typically show substantial paternal care (Smith and Wootton 1995), and male P. taeniatus occupy territories while females compete for males (Thünken et al. 2007a, 2007b). Male preference for related females might, therefore, be consistent with our model if such preference increases their female relatives' reproductive success. Additionally, $P$. taeniatus show little inbreeding depression (Thünken et al. 2007a), so selection against inbreeding preference might be weak.

Sex-specific control over mating interactions is often context dependent, for example, depending on traits expressed by individual females and males (Clutton-Brock and Parker 1995; Arnqvist and Rowe 2005). We relaxed the assumption that one sex completely controlled mating by allowing individuals of the nonchoosing sex to affect how their own quality was perceived. When females chose but males affected their own perceived quality, $S^{P}$ consistently fixed and $S^{A}$ consistently went extinct. This is because males carrying alleles that increased inbreeding $\left(S^{P}\right.$ against $S^{+}$or $S^{+}$against $\left.S^{A}\right)$ also increased their own perceived quality and, therefore, reproductive success. In contrast, when males chose but females affected their own perceived quality, end allele frequencies did not differ greatly from when females had no influence. This is because female reproductive success was not increased when females increased their own perceived quality. The way that we relaxed the assumption of complete sex-specific control over mating might or might not be biologically realistic. However, it demonstrates that complete sex-specific control over mate choice does not necessarily reflect extreme ends of the spectrum of how sexual conflict might affect inbreeding avoidance or preference.

\section{Costs}

Evolution of inbreeding strategies was strongly limited by both inbreeding depression and direct costs. Unsurprisingly, increased inbreeding depression increased the frequency of $S^{A}$ alleles and decreased the frequency of $S^{P}$ alleles. Even a small direct cost manifested as a probability of mating failure reduced $S^{A}$ and $S^{P}$ end frequencies. Quantifying both inbreeding depression and direct costs is, therefore, fundamental to predicting the evolution of biparental inbreeding strategies within and across systems but remains empirically difficult (Pomiankowski 1987; Kokko et al. 2003; Reid et al. 2015a).

Opportunity costs of male mating are high when mating with one female inhibits a male's ability to sire other females' offspring (Waser et al. 1986). When females chose, the probability that mating with a male would prevent him 
from mating with another female decreased with increasing $\Omega$, thereby decreasing male opportunity cost. In contrast, when males chose, the opportunity cost of mating was always high because mating with any one female necessarily prevented him from mating with another. This high opportunity cost is realistic for systems with substantial male parental care (Parker 1979, 2006; Waser et al. 1986), such as in species that form pair bonds (Cockburn 2006; Lukas and Clutton-Brock 2013; Szulkin et al. 2013). It is also reasonable for polygynous systems (Waser et al. 1986), such as in haremforming species where harem size is externally limited (e.g., Hurly and Robertson 1985; Ablard et al. 2013) or species where mating is limited in space (e.g., Carranza et al. 1990; Arnould and Duck 1997) or time (Yuval 2006; Diabaté et al. 2011). Even with little paternal care, mating is unlikely to be completely cost free for males. If our assumption of a high opportunity cost were strongly violated, inbreeding avoidance would be even less likely to evolve. Inbreeding tolerance might, therefore, be a common outcome when males control mating.

\section{Purging}

While rigorous theory has considered coevolution between self-fertilization and mutation load (Lande and Schemske 1985; Charlesworth and Willis 2009), no models of biparental inbreeding have incorporated analogous evolutionary feedbacks (Kokko and Ots 2006; Szulkin et al. 2013; Duthie and Reid 2015). We modeled explicit genetic architecture to provide a mechanistic basis for inbreeding depression, permitting coevolution of inbreeding strategy and mutation load. Although mutation load varied over time, it was not correlated with the frequency of $S^{P}$ or $S^{A}$ (supplemental PDF, p. S5). There was, therefore, no evidence of coevolution between inbreeding preference and mutation load and, hence, no evidence that inbreeding caused substantial purging that, in turn, facilitated further evolution of inbreeding preference.

Several factors likely impeded purging, including weak selection coefficients $(s)$ on deleterious recessive alleles and small population sizes that lead to frequent population extinction given high $s$. Additionally, modeling probabilistic survival, mate choice, and explicit relatedness structure created biologically reasonable stochasticity in the realized degrees of inbreeding and inbreeding depression. In particular, individuals carrying $S^{P}$ alleles could not inbreed with close relatives if no such relatives were available or were not selected by chance, while individuals carrying wild-type $S^{+}$ alleles sometimes inbred by chance. Such reasonable stochasticity will reduce purging given biparental inbreeding compared to that occurring given self-fertilization because individuals with alleles causing inbreeding preference are not guaranteed to inbreed.
In our model, selection coefficients against deleterious recessives never exceeded $s=0.020$, so a single locus never decreased survival probability by more than $2 \%$. Our conclusion that purging was negligible, therefore, concurs with previous models using similarly small selection coefficients. For example, Hedrick (1994) found that drift overwhelmed purging when $s<0.0624$ over $2-32$ loci, even with repeated full sibling inbreeding. Deleterious recessive alleles frequently became fixed rather than purged, often causing population extinction. Similarly, our simulations with a mutant $S^{P}$ allele greatly increased the population-wide degree of inbreeding and frequently resulted in population extinction. Extinction was, consequently, more likely given a mutant $S^{P}$ allele than a mutant $S^{A}$ allele (supplemental PDF, p. S3). A higher probability of population extinction where inbreeding preference evolves might, therefore, contribute to the rarity of inbreeding preference in nature if such populations are "evolutionary dead-ends," as suggested for self-fertilizing plants (Wright et al. 2013).

Similarly, individual-based models that examine purging when mating is random within populations (e.g., Wang et al. 1999), or random within demes connected by dispersal (e.g., Guillaume and Perrin 2006), find that the dynamics of weakly deleterious mutations are typically dominated by drift. Since inbreeding depression may commonly result from numerous weakly deleterious recessive alleles (Barrett and Charlesworth 1991; Charlesworth and Willis 2009), purging might not strongly affect the evolution of inbreeding strategies in small biparentally reproducing populations. Thus, modeling explicit mutation load might not be necessary to predict evolution of biparental inbreeding strategies. However, Wang et al. (1999) found that large-effect mutations were purged relatively effectively, implying that coevolution between biparental inbreeding strategy and mutation load cannot always be ignored.

\section{Restricted Kin Discrimination}

Inbreeding avoidance was always less likely to evolve given restricted rather than perfect kin discrimination. However, inbreeding preference was less likely to evolve given restricted kin discrimination when males chose but not when females chose. The increase in $S^{P}$ with restricted female kin discrimination arose because the quality of close kin increased relative to that of distant kin as perceived by females carrying $S^{P}$ alleles. Such females were even more likely to mate with close rather than distant male relatives that were also likely to carry the $S^{P}$ allele as well as less likely to avoid immigrant males because they were indistinguishable from distant relatives. Because immigrants carried $S^{P}$ alleles with equal frequency as the native population at arrival, immigrants were sometimes more likely to carry $S^{P}$ alleles than distant relatives. Given perfect kin discrimination, very dis- 
tant relatives might be preferred to immigrants but be less likely to carry $S^{P}$, but this is no longer the case when immigrants and distant relatives cannot be distinguished. Further exploratory simulations showed that if the assumption that immigrants carry equivalent mean allele frequencies is relaxed - for example, by assuming that immigrants never carry $S^{P}$ alleles - then restricting kin discrimination no longer facilitates evolution of inbreeding preference. Both inbreeding avoidance and inbreeding preference are, therefore, less likely to evolve when kin discrimination is restricted if such strategies are, in fact, beneficial.

\section{Conceptual Synthesis of Biparental Inbreeding Strategies}

We used a population genetic approach to examine the conditions under which selection increases the frequency of initially rare alleles that cause biparental inbreeding avoidance or inbreeding preference. Our focal question fundamentally differs from that considered by previous models of biparental inbreeding enacted though mate choice (Parker 1979, 2006; Waser et al. 1986; Lehmann and Perrin 2003; Kokko and Ots 2006; Puurtinen 2011; Lehtonen and Kokko 2015), which build from the inclusive fitness approach initiated by Parker $(1979,2006)$. Parker $(1979,2006)$ assumes that a focal individual encounters a single focal relative within a large outbred population and must decide whether to inbreed with them. By comparing the inclusive fitness consequences of inbreeding versus not, thresholds of inbreeding depression below which selection favors inbreeding can be derived. Parker $(1979,2006)$ and subsequent models show that these thresholds depend on the relatedness between two focal individuals and on the magnitude of inbreeding depression (e.g., Waser et al. 1986; Kokko and Ots 2006; Lehtonen and Kokko 2015). Such models highlight that biparental inbreeding can increase an individual's inclusive fitness even if inbreeding depression is strong, but they do not explicitly simulate allele dynamics or emerging distributions of kinship. Their approach, thereby, focuses on selection to the exclusion of other evolutionary mechanisms (Queller 2011; West and Gardner 2013). Such mechanisms include drift, which can overwhelm selection in small populations where inbreeding risk and opportunity are highest, and nonrandom mating, which can affect the distribution of kinship. By contrast, our model is not a development of Parker's $(1979,2006)$ inclusive fitness framework and does not consider pairwise encounters between two focal individuals. Instead, potential mates are encountered simultaneously, and mating behavior is defined a priori based on genotype, so no equivalents to Parker's $(1979,2006)$ inbreeding depression thresholds exist. Our approach relaxes several key assumptions of inclusive fitness models of biparental inbreeding (Duthie and Reid 2015). By explicitly tracking pedigree and allele transmission, our model integrates naturally emergent kinship distributions and evolutionary feedbacks and incorporates drift and mutation.

While the inclusive fitness and population genetic approaches differ in their aims and assumptions, there is broad conceptual overlap in predicting the consequences of selection with respect to inbreeding depression and direct negative selection on mating strategies (costs). Careful interpretation of Parker $(1979,2006)$ predicts that selection will favor inbreeding avoidance given strong inbreeding depression, while inbreeding preference should be favored for females but never males when inbreeding depression is weak (Puurtinen 2011). Accordingly, we found positive selection for alleles underlying inbreeding avoidance given sufficiently strong inbreeding depression, while positive selection for alleles underlying inbreeding preference occurred when inbreeding depression was weak but only when females also controlled mating decisions. Given female choice, it might be interesting to compare evolution of inbreeding strategy predicted from our population genetic approach to the degree of inbreeding predicted when females are able to mate optimally to maximize their inclusive fitness (Puurtinen 2011). However, such comparisons will likely require complex genetic architectures in individual-based models to allow for individual fitness optimization and will require careful consideration of analytical assumptions of optimality. As in inclusive fitness models (e.g., Waser et al. 1986; Lehmann and Perrin 2003), direct costs in our model often outweighed the benefits of an active inbreeding strategy, causing selection against inbreeding avoidance or inbreeding preference. Tolerance of biparental inbreeding, manifested as random mating with respect to relatedness, might, therefore, be the most likely outcome under wide-ranging conditions.

We modeled inbreeding strategy in the context of mate choice through kin discrimination, not in the context of dispersal, as is the focus of other genetically explicit models (e.g., Perrin and Mazalov 2000; Roze and Rousset 2005, 2009; Guillaume and Perrin 2006, 2009). Such models show that selection for inbreeding avoidance can contribute to evolution of dispersal, but optimal dispersal rates are also predicted to be greatly influenced by their effect on reducing kin competition (Guillaume and Perrin 2006; Roze and Rousset 2009). Our results highlight conceptual overlap between inbreeding avoidance enacted through mate choice versus dispersal. For example, Roze and Rousset (2009) found that higher dispersal rates evolved given increasingly strong inbreeding depression in offspring of philopatric individuals, but selection for high dispersal rate was also inhibited by direct costs. Further, lower dispersal rates evolved in larger demes due to the decreased risk of inbreeding (Roze and Rousset 2009). Our results, therefore, suggest that adaptive evolution of inbreeding strategy is likely restricted to small or viscous populations regardless of the mechanism by which inbreeding strategy is enacted. 


\section{Acknowledgments}

This work was funded by a European Research Council Consolidator Grant and a Royal Society University Research Fellowship to J.M.R. We thank G. Bocedi, L. Keller, H. Kokko, S. Losdat, and M. Wolak for their helpful comments. Computer simulations were performed using the Maxwell Computing Cluster at the University of Aberdeen.

\section{Literature Cited}

Ablard, K. M., P. W. Schaefer, and G. Gries. 2013. An alternative reproductive tactic: a parasitoid wasp gathers and guards a harem by pheromone-tagging virgins. Behavioural Processes 94:32-40.

Angeloni, F., N. J. Ouborg, and R. Leimu. 1998. Reproductive strategies of yellow-bellied marmots: energy conservation and differences between the sexes. Journal of Mammalogy 79:385-393.

- 2003. Reproductive competition in female yellow-bellied marmots. Pages 133-142 in R. Ramousse, D. Allaine, and M. Le Berre, eds. Adaptive strategies and diversity in marmots, Armitage 1986. International Marmot Network, Lyon.

2011. Meta-analysis on the association of population size and life history with inbreeding depression in plants. Biological Conservation 144:35-43.

Arnould, J., and C. Duck. 1997. The cost and benefits of territorial tenure, and factors affecting mating success in male Antarctic fur seals. Journal of Zoology 241:649-664.

Arnqvist, G., and L. Rowe. 2005. Sexual conflict. Princeton University Press, Princeton, NJ.

Banks, S. C., and D. B. Lindenmayer. 2013. Inbreeding avoidance, patch isolation and matrix permeability influence dispersal and settlement choices by male agile antechinus in a fragmented landscape. Journal of Animal Ecology 83:515-524.

Barrett, S. C. H., and D. Charlesworth. 1991. Effects of a change in the level of inbreeding on genetic load. Nature 352:522-524.

Benesh, D. P., F. Weinreich, M. Kalbe, and M. Milinski. 2014. Lifetime inbreeding depression, purging, and mating system evolution in a simultaneous hermaphrodite tapeworm. Evolution 68:17621774 .

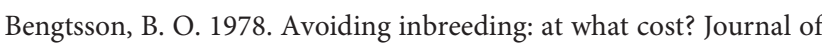
Theoretical Biology 73:439-444.

Boyce, A. 1983. Computation of inbreeding and kinship coefficients on extended pedigrees. Journal of Heredity 74:400-404.

Brouwer, L., M. van de Pol, E. Atema, and A. Cockburn. 2011. Strategic promiscuity helps avoid inbreeding at multiple levels in a cooperative breeder where both sexes are philopatric. Molecular Ecology 20:4796-4807.

Caballero, A. 1994. Developments in the prediction of effective population size. Heredity 73:657-679.

Carranza, J., F. Alvarez, and T. Redondo. 1990. Territoriality as a mating strategy in red deer. Animal Behaviour 40:79-88.

Charlesworth, B., and D. Charlesworth. 1999. The genetic basis of inbreeding depression. Genetical Research 74:329-340.

Charlesworth, D. 2006. Evolution of plant breeding systems. Current Biology 16:R726-R735.

Charlesworth, D., and B. Charlesworth. 1987. Inbreeding depression and its evolutionary consequences. Annual Review of Ecology and Systematics 18:237-268.
Charlesworth, D., M. T. Morgan, and B. Charlesworth. 1990. Inbreeding depression, genetic load, and the evolution of outcrossing rates in a multilocus system with no linkage. Evolution 44:1469-1489.

Charlesworth, D., and J. H. Willis. 2009. The genetics of inbreeding depression. Nature Reviews Genetics 10:783-796.

Cheptou, P.-O., and K. Donohue. 2011. Environment-dependent inbreeding depression: its ecological and evolutionary significance. New Phytologist 189:395-407.

Clutton-Brock, T., and G. A. Parker. 1995. Sexual coercion in animal societies. Animal Behaviour 49:1345-1365.

Cockburn, A. 2006. Prevalence of different modes of parental care in birds. Proceedings of the Roval Societv B 273:1375-1383.

Cohen, L. B., and D. C. Dearborn. 2004. Great frigatebirds, Fregata minor, choose mates that are genetically similar. Animal Behaviour 68:1229-1236.

Crnokrak, P., and S. C. H. Barrett. 2002. Perspective: purging the genetic load: a review of the experimental evidence. Evolution 56:23472358.

Diabaté, A., A. S. Yaro, A. Dao, M. Diallo, D. L. Huestis, and T. Lehmann. 2011. Spatial distribution and male mating success of Anopheles gambiae swarms. BMC Evolutionary Biology 11:184.

Dudley, S. A., and A. L. File. 2007. Kin recognition in an annual plant. Biology Letters 3:435-438.

Duthie, A. B., and J. M. Reid. 2015. What happens after inbreeding avoidance? inbreeding by rejected relatives and the inclusive fitness benefit of inbreeding avoidance. PLoS ONE 10:e0125140.

2016. Data from: Evolution of inbreeding avoidance and inbreeding preference through mate choice among interacting relatives. American Naturalist, Dryad Digital Repository, http://dx.doi.org/10 $.5061 /$ dryad.3861f.

Fisher, R. A. 1941. Average excess and average effect of a gene substitution. Annals of Eugenics 11:53-63.

. 1949. The theory of inbreeding. Oliver and Boyd, Edinburgh. Frankham, R., D. Gilligan, D. Morris, and D. Briscoe. 2001. Inbreeding and extinction: effects of purging. Conservation Genetics 2:279285.

Frommen, J. G., and T. C. M. Bakker. 2006. Inbreeding avoidance through non-random mating in sticklebacks. Biology Letters 2:232235.

Gandon, S. 1999. Kin competition, the cost of inbreeding and the evolution of dispersal. Journal of Theoretical Biology 200:345-364.

Geffen, E., M. Kam, R. Hefner, P. Hersteinsson, A. Angerbjörn, L. Dalèn, E. Fuglei, et al. 2011. Kin encounter rate and inbreeding avoidance in canids. Molecular Ecology 20:5348-5358.

Goodwillie, C., S. Kalisz, and C. G. Eckert. 2005. The evolutionary enigma of mixed mating systems in plants: occurrence, theoretical explanations, and empirical evidence. Annual Review of Ecology, Evolution, and Systematics 36:47-79.

Griffin, A., and S. West. 2003. Kin discrimination and the benefit of helping in cooperatively breeding vertebrates. Science 302:634-637.

Guillaume, F., and N. Perrin. 2006. Joint evolution of dispersal and inbreeding load. Genetics 173:497-509.

2009. Inbreeding load, bet hedging, and the evolution of sexbiased dispersal. American Naturalist 173:536-541.

Hansson, B., L. Jack, J. K. Christians, J. M. Pemberton, M. Akesson, H. Westerdahl, S. Bensch, and D. Hasselquist. 2006. No evidence for inbreeding avoidance in a great reed warbler population. Behavioral Ecology 18:157-164.

Hedrick, P. W. 1994. Purging inbreeding depression and the probability of extinction: full-sib mating. Heredity 73:363-372. 
Herfindal, I., and H. Haanes. 2014. Population properties affect inbreeding avoidance in moose. Biology Letters 10:20140786.

Hurly, T. A., and R. J. Robertson. 1985. Do female red-winged blackbirds limit harem size? I. A removal experiment. Auk 102:205-209.

Jamieson, I. G., S. S. Taylor, L. N. Tracy, H. Kokko, and D. P. Armstrong. 2009. Why some species of birds do not avoid inbreeding: insights from New Zealand robins and saddlebacks. Behavioral Ecology 20:575-584.

Karron, J. D., C. T. Ivey, R. J. Mitchell, M. R. Whitehead, R. Peakall, and A. L. Case. 2012. New perspectives on the evolution of plant mating systems. Annals of Botany 109:493-503.

Keane, B., S. R. Creel, and P. M. Waser. 1996. No evidence of inbreeding avoidance or inbreeding depression in a social carnivore. Behavioral Ecology 7:480-489.

Keller, L. F., and P. Arcese. 1998. No evidence for inbreeding avoidance in a natural population of song sparrows (Melospiza melodia). American Naturalist 152:380-392.

Keller, L. F., and D. M. Waller. 2002. Inbreeding effects in wild populations. Trends in Ecology and Evolution 17:19-23.

Kingma, S. A., M. L. Hall, and A. Peters. 2013. Breeding synchronization facilitates extrapair mating for inbreeding avoidance. Behavioral Ecology 24:1390-1397.

Kleven, O., F. Jacobsen, R. J. Robertson, and J. T. Lifjeld. 2005. Extrapair mating between relatives in the barn swallow: a role for kin selection? Biology Letters 1:389-392.

Kokko, H., R. Brooks, M. D. Jennions, and J. Morley. 2003. The evolution of mate choice and mating biases. Proceedings of the Roval Society B 270:653-664.

Kokko, H., and I. Ots. 2006. When not to avoid inbreeding. Evolution 60:467-475

Lande, R., and D. W. Schemske. 1985. The evolution of self-fertilization and inbreeding depression in plants. I. Genetic models. Evolution 39: $24-40$.

Langen, K., J. Schwarzer, H. Kullmann, T. C. M. Bakker, and T. Thünken. 2011. Microsatellite support for active inbreeding in a cichlid fish. PLoS ONE 6:e24689.

Latta, R., and K. Ritland. 1993. Models for the evolution of selfing under alternative modes of inheritance. Heredity 71:1-10.

Lawson Handley, L. J., and N. Perrin. 2007. Advances in our understanding of mammalian sex-biased dispersal. Molecular Ecology 16:1559-1578.

Lehmann, L., and N. Perrin. 2003. Inbreeding avoidance through kin recognition: choosy females boost male dispersal. American Naturalist 162:638-652.

Lehtonen, J., and H. Kokko. 2015. Why inclusive fitness can make it adaptive to produce less fit extra-pair offspring. Proceedings of the Roval Society B 282:20142716.

Lemâttre, J.-F., S. A. Ramm, J. L. Hurst, and P. Stockley. 2012. Inbreeding avoidance behaviour of male bank voles in relation to social status. Animal Behaviour 83:453-457.

Lloyd, D. 1992. Self-and cross-fertilization in plants. II. The selection of self-fertilization. International Journal of Plant Sciences 153:370-380.

Lukas, D., and T. H. Clutton-Brock. 2013. The evolution of social monogamy in mammals. Science 341:526-530.

Lynch, M., and B. Walsh. 1998. Genetics and analysis of quantitative traits. Sinauer, Sunderland, MA.

Manly, B. F. J. 2007. Randomization, bootstrap and Monte Carlo methods in biology. 3rd ed. Chapman and Hall, Boca Raton, FL.

Mateo, J. M. 2002. Kin-recognition abilities and nepotism as a function of sociality. Proceedings of the Roval Society B 269:721-727.
Mettler, L. E., and T. G. Gregg. 1969. Population genetics and evolution. Prentice Hall, Eaglewood Cliffs, NJ.

Mills, L. S., and P. E. Smouse. 1994. Demographic consequences of inbreeding in remnant populations. American Naturalist 144:412431.

Morton, N. E., J. F. Crow, and H. J. Muller. 1956. An estimate of the mutational damage in man from data on consanguineous marriages. Proceedings of the National Academv of Sciences of the USA 42:855863.

Nelson, J. B. 1975. The breeding biology of frigatebirds: a comparative review. Living Bird 14:113-155.

Nichols, H. J., M. A. Cant, J. I. Hoffman, and J. L. Sanderson. 2014 Evidence for frequent incest in a cooperatively breeding mammal. Biology Letters 10:20140898.

O'Grady, J. J., B. W. Brook, D. H. Reed, J. D. Ballou, D. W. Tonkyn, and R. Frankham. 2006. Realistic levels of inbreeding depression strongly affect extinction risk in wild populations. Biological Conservation 133:42-51.

Olofsson, H., A. M. Frame, and M. R. Servedio. 2011. Can reinforcement occur with a learned trait? Evolution 65:1992-2003.

Olson, L. E., D. T. Blumstein, J. R. Pollinger, and R. K. Wayne. 2012. No evidence of inbreeding avoidance despite demonstrated survival costs in a polygynous rodent. Molecular Ecology 21:562-571.

Parker, G. A. 1979. Sexual selection and sexual conflict. Pages 123-166 in M. S. Blum and N. A. Blum, eds. Sexual selection and reproductive competition in insects. Academic Press, New York.

2006. Sexual conflict over mating and fertilization: an overview. Philosophical Transactions of the Roval Society B 361:235259.

Perrin, N., and V. Mazalov. 1999. Dispersal and inbreeding avoidance. American Naturalist 154:282-292.

2000. Local competition, inbreeding, and the evolution of sex-biased dispersal. American Naturalist 155:116-127.

Pizzari, T., J. M. Biernaskie, and P. Carazo. 2015. Inclusive fitness and sexual conflict: how population structure can modulate the battle of the sexes. BioEssavs 37:155-166.

Pizzari, T., and A. Gardner. 2012. The sociobiology of sex: inclusive fitness consequences of inter-sexual interactions. Philosophical Transactions of the Roval Society B 367:2314-2323.

Pizzari, T., H. Løvlie, and C. K. Cornwallis. 2004. Sex-specific, counteracting responses to inbreeding in a bird. Proceedings of the Roval Societv B 271:2115-2121.

Pomiankowski, A. 1987. The costs of choice in sexual selection. Journal of Theoretical Biology 128:195-218.

Pomiankowski, A., Y. Iwasa, and S. Nee. 1991. The evolution of costly mate preferences I. Fisher and biased mutation. Evolution 45:14221430.

Porcher, E., and R. Lande. 2005. The evolution of self-fertilization and inbreeding depression under pollen discounting and pollen limitation. Journal of Evolutionary Biology 18:497-508.

2013. Evaluating a simple approximation to modeling the joint evolution of self-fertilization and inbreeding depression. Evolution 67:3628-3635.

Proulx, S. R., and M. R. Servedio. 2009. Dissecting selection on female mating preferences during secondary contact. Evolution 63: 2031-2046.

Pusey, A., and M. Wolf. 1996. Inbreeding avoidance in animals. Trends in Ecology and Evolution 5347:298-301.

Pusey, A. E. 1987. Sex-biased dispersal and inbreeding avoidance in birds and mammals. Trends in Ecology and Evolution 2:295-299. 
Puurtinen, M. 2011. Mate choice for optimal (k)inbreeding. Evolution 65:1501-1505.

Queller, D. C. 1994. Genetic relatedness in viscous populations. Evolutionary Ecology 7653:70-73.

. 2011. Expanded social fitness and Hamilton's rule for kin, kith, and kind. Proceedings of the National Academy of Sciences of the USA 108:10792-10799.

Ralls, K., J. D. Ballou, and A. Templeton. 1988. Estimates of lethal equivalents and the cost of inbreeding in mammals. Conservation Biology 2:185-193.

Reid, J. M., P. Arcese, L. F. Keller, R. R. Germain, A. B. Duthie, S. Losdat, M. E. Wolak, and P. Nietlisbach. 2015. Quantifying inbreeding avoidance through extra-pair reproduction. Evolution 69 : 59-74.

Reynolds, S. M., J. A. C. Uy, G. L. Patricelli, S. W. Coleman, M. J. Braun, and G. Borgia. 2014. Tests of the kin selection model of mate choice and inbreeding avoidance in satin bowerbirds. Behavioral Ecology 25:1005-1014.

Rowe, L., G. Arnqvist, A. Sih, and J. J. Krupa. 1994. Sexual conflict and the evolutionary ecology of mating patterns: water striders as a model system. Trends in Ecology and Evolution 9:289-293.

Roze, D., and F. Rousset. 2005. Inbreeding depression and the evolution of dispersal rates: a multilocus model. American Naturalist $166: 708-721$

. 2009. Strong effects of heterosis on the evolution of dispersal rates. Journal of Evolutionary Biology 22:1221-1233.

Smith, C., and R. J. Wootton. 1995. The costs of parental care in teleost fishes. Reviews in Fish Biology and Fisheries 22:7-22.

Strassmann, J. E., O. M. Gilbert, and D. C. Queller. 2011. Kin discrimination and cooperation in microbes. Annual Review of Microbiology 65:349-367.

Szulkin, M., K. V. Stopher, J. M. Pemberton, and J. M. Reid. 2013. Inbreeding avoidance, tolerance, or preference in animals? Trends in Ecology and Evolution 28:205-211.

Tennenhouse, E. M. 2014. Inbreeding avoidance in male primates: a response to female mate choice? Ethology 120:111-119.

Theodorou, K., and D. Couvet. 2006. On the expected relationship between inbreeding, fitness, and extinction. Genetics Selection Evolution 38:371-387.

Thünken, T., T. C. M. Bakker, and S. A. Baldauf. 2013. "Armpit effect" in an African cichlid fish: self-referent kin recognition in mating decisions of male Pelvicachromis taeniatus. Behavioral Ecology and Sociobiology 68:99-104.

Thünken, T., T. C. M. Bakker, S. A. Baldauf, and H. Kullmann. $2007 a$. Active inbreeding in a cichlid fish and its adaptive significance. Current Biology 17:225-229. 2007b. Direct familiarity does not alter mating preference for sisters in male Pelvicachromis taeniatus (Cichlidae). Ethology 113:1107-1112.

Travis, J. M. J., and T. H. G. Ezard. 2006. Habitat geometry, population viscosity and the rate of genetic drift. Ecological Informatics $1: 153-161$

Tregenza, T., and N. Wedell. 2002. Polyandrous females avoid costs of inbreeding. Nature 415:71-73.

Tregenza, T., N. Wedell, and T. Chapman. 2006. Introduction. Sexual conflict: a new paradigm? Philosophical Transactions of the Roval Society B 361:229-234.

Turner, M. 1982. Homozygosity and patch structure in plant populations as a result of nearest-neighbor pollination. Proceedings of the National Academv of Sciences of the USA 79:203-207.

Verzijden, M. N., R. F. Lachlan, and M. R. Servedio. 2005. Female matechoice behavior and sympatric speciation. Evolution 59:2097-2108.

Wang, C., and X. Lu. 2011. Female ground tits prefer relatives as extrapair partners: driven by kin-selection? Molecular Ecology 20:28512863.

Wang, J., W. G. Hill, D. Charlesworth, and B. Charlesworth. 1999. Dynamics of inbreeding depression due to deleterious mutations in small populations: mutation parameters and inbreeding rate. Genetical Research 74:165-178.

Waser, P. M., S. N. Austad, and B. Keane. 1986. When should animals tolerate inbreeding? American Naturalist 128:529-537.

West, S. A., and A. Gardner. 2013. Adaptation and inclusive fitness. Current Biology 23:R577-R584.

White, J. W., A. Rassweiler, J. F. Samhouri, A. C. Stier, and C. White. 2014. Ecologists should not use statistical significance tests to interpret simulation model results. Oikos 123:385-388.

Wright, S. 1931.Evolution in Mendelian populations. Genetics 16:97-159.

Wright, S. I., S. Kalisz, and T. Slotte. 2013. Evolutionary consequences of self-fertilization in plants. Proceedings of the Roval Societv B 280: 20130133.

Young, A. G., L. M. Broadhurst, and P. H. Thrall. 2012. Non-additive effects of pollen limitation and self-incompatibility reduce plant reproductive success and population viability. Annals of Botany 109: 643-653.

Yuval, B. 2006. Mating systems of blood-feeding flies. Annual Review of Entomology 51:413-40.

Zimmer, S. M., H. Krehenwinkel, and J. M. Schneider. 2014. Rapid range expansion is not restricted by inbreeding in a sexually cannibalistic spider. PLoS ONE 9:e95963.

Associate Editor: G. Sander van Doorn Editor: Yannis Michalakis 